\title{
Step-by-step control of target efficiency indices of the control and measuring equipment stock applied in construction and housing and communal services
}

\author{
Rustam Khayrullin ${ }^{l, *}$, and Pavel Ivanov ${ }^{l}$ \\ ${ }^{1}$ Moscow State University of Civil Engineering, Yaroslavskoe shosse, 26, Moscow, 129337, Russia
}

\begin{abstract}
The mathematical model is considered for the formation and implementation of development strategies of the stock of control and measuring instruments (CMI) applied in construction and housing and communal services(HCS), and step-by-step control of efficiency target values of the stock. The model is based on a system of finite - difference equations describing the change of number of the CMI samples with different levels of technical perfection and technical condition at each planning interval. The model allows calculating the required number of $\mathrm{CMI}$ for procurement and repairs in the various groups for provide target values of efficiency indices at each planning interval. Controller is number of modern CMI samples for procurement and number of modern and obsolete faulty CMI samples for the repairs. The results of calculations are presented.
\end{abstract}

\section{Description of the set of possible states of $\mathrm{CMI}$ samples and possible transitions}

Control of the modernity and serviceability indices of the stock of control and measuring instrument $(\mathrm{CMI})$ is an actual practical task in the spheres of housing and communal services(HCS) [1,2], construction [3] and military [4,5].

We assume, that CMI samples, according to the levels of technical perfection and technical condition, are subdivided into modern serviceable, modern faulty, obsolete serviceable and obsolete faulty. We denote by $N=x_{1}+x_{2}+x_{3}+x_{4}$ - total number of CMI samples, when $x_{1}$ - number of modern serviceable CMI samples, $x_{2}$ - number of modern faulty CMI samples, $x_{3}$ - number of obsolete serviceable CMI samples, $x_{4}$ - number of obsolete faulty CMI samples. The total number of CMI samples in the stock is determined by the supply standards [1], as well as tactical and strategic tasks assigned to the management companies (HCS services, metrology units).

Let by means of statistical processing of data for a sufficiently long period of time, the following "statistical" probabilities of state transitions of CMI samples is determined:

$p_{12}, p_{13}$ - the probability of a transition from modern serviceable to modern faulty, obsolete serviceable, respectively; 
$p_{24}$ - the probability of transition from a modern faulty state to an obsolete faulty state;

$p_{34}$ - the probability of a transition from the obsolete serviceable state to the obsolete faulty state.

We describe other transitions of states, which in the present paper are considered as controller: $u_{21}$ - repair of modern faulty samples, $u_{43}$ - repair of obsolete faulty samples, $u_{4}$ - procurement of modern serviceable samples, $u_{6}$ - discarding of obsolete faulty samples.

The graph of state transitions is shown in Fig. 1.

The dynamic equations for the development of the CMI stock have

$$
\left\{\begin{array}{l}
\frac{d x_{1}}{d t}=-p_{12} x_{1}-p_{13} x_{1}+u_{21}+u_{4} \\
\frac{d x_{2}}{d t}=-p_{24} x_{2}+p_{12} x_{1}-u_{21} \\
\frac{d x_{3}}{d t}=-p_{34} x_{3}+p_{13} x_{1}+u_{43} \\
\frac{d x_{4}}{d t}=p_{24} x_{2}+p_{34} x_{3}-u_{43}-u_{6}
\end{array}\right.
$$

The initial conditions are assumed to be given:

$$
x_{i}(0)=x_{i}^{\text {sad }},(i=1,2,3,4)
$$

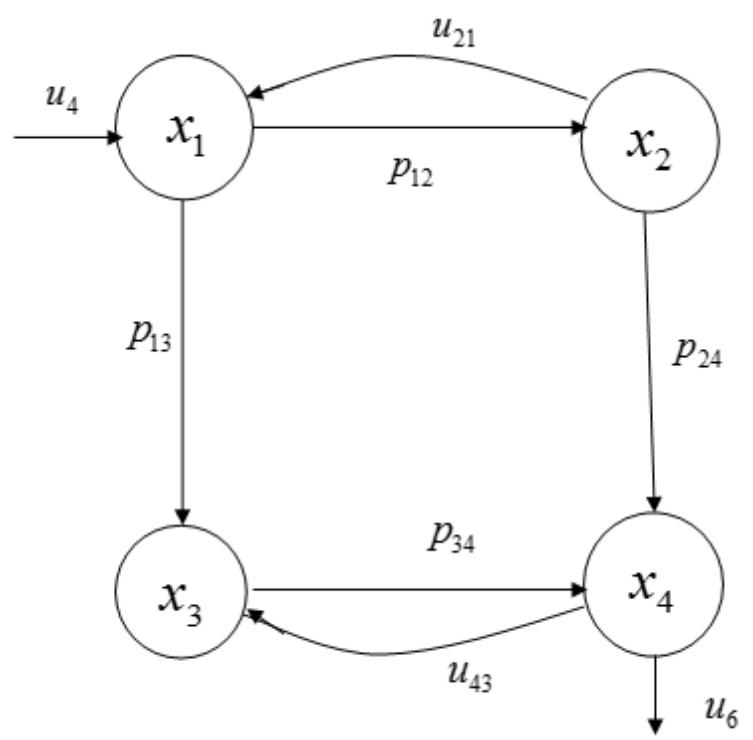

Fig. 1. Graph of state transitions of CMI.

\section{Statement of the problem of step-by-step control of the target efficiency indices of the CMI stock}


For a given initial state of the CMI stock (a given number of CMI samples having different levels of technical perfection and condition), it is required to formation strategies for controlling procurement and repairs of CMI samples so that supply standards and target values of efficiency indices (modernity and serviceability) were satisfied at the end of the planned period taking into account the dynamics of the state transitions of the CMI samples during the planning period. In addition, these indices and the total number of CMI samples in the stock is should change strictly evenly from the beginning to the end of planning. At the same time, the total number of CMI samples at the end of the planning period is determined by the supply standards [1] and the target values of performance indicators - by the directives, as well as the strategic and tactical tasks assigned to the metrological services of the CMI provision.

\section{Step-by-step solution of the system of development dynamics equations of the $\mathrm{CMI}$ stock}

The planning period is divided into planning intervals having a duration of one year.

At each planning step $t=\overline{1, T}$, the total number of CMI samples is determined,

$$
x_{1}(t)+x_{3}(t)+x_{3}(t)+x_{4}(t)=N(t) .
$$

The number of modern samples, the number of serviceable samples and the increase in the total number of CMI:

$$
\left\{\begin{array}{l}
x_{1}(t)+x_{2}(t)=N(t) P_{c}(t) \\
x_{1}(t)+x_{3}(t)=N(t) P_{u}(t) \\
u_{4}(t)-u_{6}(t)=C_{3}(t)
\end{array}\right.
$$

when $C_{3}(t)$ - the target value of the increase in the total number of CMI samples, $P_{c}(t)$, $P_{u}(t)$ - target values of the indices of modernity and serviceability on the interval $t$.

The required values of the target parameters are also determined at the end of the current planning interval (the beginning of the next planning interval): $C_{3}(t+1), P_{c}(t+1)$, $P_{u}(t+1), N(t+1)$.

Step-by-step solution of system (1) with initial conditions (2) has of the form:

$$
\left\{\begin{array}{l}
x_{1}(t+1)=x_{1}(t)-p_{12} x_{1}(t)-p_{13} x_{1}(t)+u_{21}(t)+u_{4}(t) \\
x_{2}(t+1)=x_{2}(t)-p_{24} x_{2}(t)+p_{12} x_{1}(t)-u_{21}(t) \\
x_{3}(t+1)=x_{3}(t)-p_{34} x_{3}(t)+p_{13} x_{1}(t)+u_{43}(t) \\
x_{4}(t+1)=x_{4}(t)+p_{24} x_{2}(t)+p_{34} x_{3}(t)-u_{43}(t)-u_{6}(t)
\end{array},\right.
$$

Consider the first case $u_{43}(t)=0$, corresponding to the strategy, when only modern faulty CMI samples are should repaired. Then the system of equations (3) can be represented in the form:

$$
\left\{\begin{array}{l}
x_{1}(t)+x_{2}(t)-p_{13} x_{1}(t)-p_{24} x_{2}(t)+u_{4}(t)=N(t+1) P_{c}(t+1) \\
x_{1}(t)+x_{3}(t)-p_{12} x_{1}(t)-p_{34} x_{3}(t)+u_{21}(t)+u_{4}(t)=N(t+1) P_{u}(t+1), \\
u_{4}(t)-u_{6}(t)=C_{3}(t+1)
\end{array}\right.
$$

Considering these relations as a system of linear algebraic equations with respect to variables $u_{21}(t), u_{4}(t), u_{6}(t)$. We note that the system has a nonzero determinant. Therefore, there exists a unique solution of the system, which can be represented in the form: 


$$
\left\{\begin{array}{l}
u_{4}(t)=N(t+1) P_{c}(t+1)-x_{1}(t)-x_{2}(t)+p_{13} x_{1}(t)+p_{24} x_{2}(t) \\
u_{21}(t)=N(t+1)\left(P_{u}(t+1)-P_{c}(t)\right)-x_{3}(t)+x_{2}(t)+p_{12} x_{1}(t)+p_{34} x_{3}(t)-p_{13} x_{1}(t)-p_{24} x_{2}(t), \\
u_{6}(t)=N(t+1) P_{c}(t+1)-x_{1}(t)-x_{2}(t)+p_{13} x_{1}(t)+p_{24} x_{2}(t)-C_{3}(t+1)
\end{array}\right.
$$

Calculations showed that to satisfy the targets for most planning intervals, it is sufficient to use as control parameters only procurement and repairs of modern faulty samples.

At the same time, the unique solution (in accordance with the theory of systems of linear equations) $u_{21}(t), u_{4}(t), u_{6}(t)$, can be negative for one or several variables, which leads to the impossibility of its practical implementation.

This circumstance indicates that the resource used for control is not sufficient to simultaneously satisfaction the requirements of modernity, serviceability and availability. In this case, to obtain a result, usable for practical, it is necessary to attract additional resources. For example, repairing obsolete faulty samples or jointly performing repairs of both modern faulty and obsolete faulty CMI samples. One of these possibilities is described below.

Let, for example, for a certain value of $t$ equal $x_{4}(t)<0$. In this case, we will expand the control capabilities and allow the possibility of repairing obsolete faulty samples: $u_{43}(t) \neq 0$. We obtain

$$
\left\{\begin{array}{l}
x_{1}(t+1)=x_{1}(t)-p_{12} x_{1}(t)-p_{13} x_{1}(t)+u_{21}(t)+u_{4}(t) \\
x_{2}(t+1)=x_{2}(t)-p_{24} x_{2}(t)+p_{12} x_{1}(t)-u_{21}(t) \\
x_{3}(t+1)=x_{3}(t)-p_{34} x_{3}(t)+p_{13} x_{1}(t)+u_{43}(t)+u_{43}(t) \\
x_{4}(t+1)=x_{4}(t)+p_{24} x_{2}(t)+p_{34} x_{3}(t)-u_{43}(t)-u_{6}(t)
\end{array},\right.
$$

Equations (3) can be rewritten as

$$
\left\{\begin{array}{l}
x_{1}(t)+x_{2}(t)-p_{13} x_{1}(t)-p_{24} x_{2}(t)+u_{4}(t)=N(t+1) P_{c}(t+1) \\
x_{1}(t)+x_{3}(t)-p_{12} x_{1}(t)-p_{34} x_{3}(t)+u_{21}(t)+u_{4}(t)+u_{43}(t)=N(t+1) P_{u}(t+1), \\
u_{4}(t)-u_{6}(t)=C_{3}(t+1)
\end{array}\right.
$$

Note that the number of system variables is greater than the number of equations. In the general case, the system has an infinite number of solutions. Control will be chosen so that the number of modern faulty samples in each planning interval is zero (all modern faulty CMI samples are to be repaired in the same planning interval on which they became faulty):

$$
u_{21}(t)=x_{2}(t)-p_{24} x_{2}(t)+p_{12} x_{1}(t)
$$

Then the remaining controls are uniquely:

$$
\left\{\begin{array}{l}
u_{4}(t)=N(t+1) P_{C}(t+1)-x_{1}(t)-x_{2}(t)+p_{13} x_{1}(t)+p_{24} x_{2}(t) \\
u_{43}(t)=N(t+1)\left(P_{u}(t+1)-P_{C}(t+1)\right)-x_{3}(t)+p_{34} x_{3}(t)-p_{13} x_{1}(t) \\
u_{6}(t)=N(t+1) P_{C}(t+1)-x_{1}(t)-x_{2}(t)+p_{13} x_{1}(t)+p_{24} x_{2}(t)-C_{3}(t+1)
\end{array},\right.
$$

Calculations have shown that when combining planning intervals containing and, are possible both non-negative solutions of the system and step-by-step practical implementation of target values and supply standards.

\section{Results of calculations}

The calculations were carried out for the target values of the indices of the modernity and the serviceability of 0.7 and 0.8 , respectively. At the same time, it is required in accordance with the target supply standards for the planning period to increase the total number of CMI samples by one and a half times (this is highlighted in the last line of Table 1, italics). The rest of the original data is contained in the second line of Table 1 (italics). 
Table 1 shows the dynamics in the number of CMI samples of different levels of technical perfection and technical condition, as well as the number of procurement and repaired CMI samples. The total number of CMI samples as well as the indices of modernity and serviceability vary uniformly from the initial values to the target values. It can be seen that for the step-by-step satisfaction of the indices, it is sufficient to procurement, the write-offs and repairs of modern faulty CMI samples, with the exception of the 6th and 7th year of planning. At these intervals, it is necessary to additionally repair obsolete faulty samples. At 8-10 scheduling intervals, it is possible to confine oneself to repairing only modern faulty samples.

Table 1. Development dynamics of the CMI stock.

\begin{tabular}{|c|c|c|c|c|c|c|c|c|c|c|c|}
\hline Year & $\mathrm{N}$ & Х1 & Х2 & Х3 & Х4 & U4 & U6 & U21 & U43 & Рс & Ри \\
\hline 0 & $\mathbf{1 0 0 0 0}$ & $\mathbf{5 0 0 0}$ & $\mathbf{1 0 0 0}$ & $\mathbf{2 0 0 0}$ & $\mathbf{2 ~ 0 0 0}$ & 725 & 225 & 580 & 0 & $\mathbf{0 , 6 0}$ & $\mathbf{0 , 7 0}$ \\
\hline 1 & 10500 & 5805 & 600 & 1650 & 2445 & 747 & 247 & 503 & 0 & 0,61 & 0,71 \\
\hline 2 & 11000 & 6475 & 345 & 1445 & 2735 & 773 & 273 & 459 & 0 & 0,62 & 0,72 \\
\hline 3 & 11500 & 7060 & 185 & 1335 & 2920 & 801 & 301 & 438 & 0 & 0,63 & 0,73 \\
\hline 4 & 12000 & 7592 & 88 & 1288 & 3032 & 831 & 331 & 430 & 0 & 0,64 & 0,74 \\
\hline $\mathbf{5}$ & 12500 & 8094 & 31 & 1281 & 3094 & 862 & 362 & 432 & 0 & 0,65 & 0,75 \\
\hline 6 & 13000 & 8579 & 1 & 1301 & 3119 & 894 & 394 & 430 & 10 & 0,66 & 0,76 \\
\hline 7 & 13500 & 9045 & 0 & 1350 & 3105 & 927 & 427 & 452 & 3 & 0,67 & 0,77 \\
\hline 8 & 14000 & 9520 & 0 & 1400 & 3080 & 961 & 461 & 470 & 0 & 0,68 & 0,78 \\
\hline 9 & 14500 & 9999 & 6 & 1456 & 3039 & 995 & 495 & 486 & 0 & 0,69 & 0,79 \\
\hline 10 & $\mathbf{1 5 0 0 0}$ & 10481 & 19 & 1519 & 2981 & 1030 & 530 & 504 & 0 & $\mathbf{0 , 7 0}$ & $\mathbf{0 , 8 0}$ \\
\hline
\end{tabular}

In Fig. 2, the indices of serviceability and modernity for a development strategy with "uniform" procurement and repairs are depicted. It can be seen that the efficiency indices are nonlinear; they reach target values with some ahead [6]. As a result, at the time of the end of the planning, the CMI stock will have another structure: another number of modern and serviceable CMI samples.

Because of such non-uniformity, the CMI stock, which has the same modernity and serviceability indices at the end of planning, turns out to be more "obsolete", which in the future will lead to the need for more aggressive control of procurement and repairs in the subsequent period stages of long-term planning.

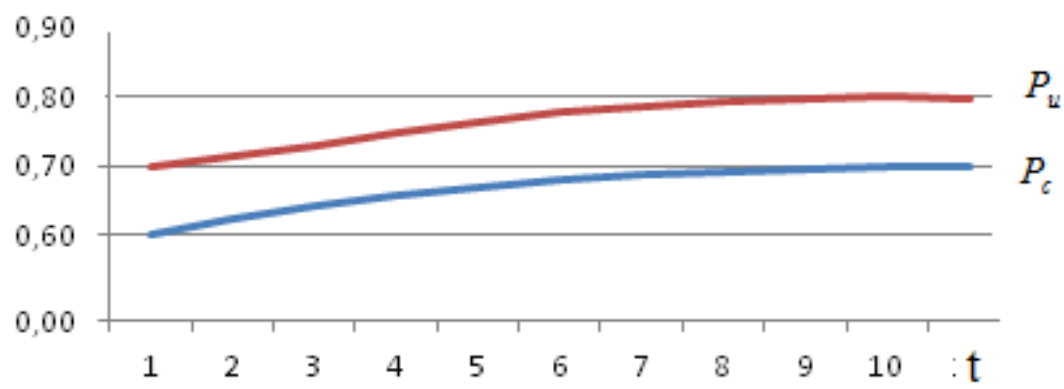

Fig. 2. Dynamics of modernity and serviceability indices of the CMI stock

\section{Conclusion.}


The main results of the work are as follows:

1. A finite-difference dynamic model was developed for step-by-step control of the target efficiency indices of the CMI stock.

2. Algorithms for calculating control parameters providing the target indices of development of the CMI stock.

3. Programmatically obtained the solution of the problem - target control of the efficiency indices of the CMI stock in the form of synthesis. The explicit formulas are derived, allowing calculating the values of the control parameters necessary to satisfy the target values of the indices of the procurement, serviceability and availability.

\section{References}

1. N.Ju. Jas'kova, D.M. Karasik, Vestnik MGSU, 9, 182 (2014)

2. R.Z. Khayrullin, MATEC Web Conf., 117, 76 (2017)

3. I.S. Lifanov, N.G. Sherstjukov, Metrology, tools and methods of quality control in construction (Stroyizdat, Moscow, 1979)

4. V.M. Burenok, R.N. Pogrebnjak, A.P. Skotnikov, Methodology for substantiating the prospects for the development of general-purpose weapons (Machine Building, Moscow, 2010)

5. A.N. D'jakov, D.V. Reshetnikov, S.N. Bojarshinov, Armament and economics, 36, 35 (2016)

6. P.A. Marichev, A.S. Kornev, R.Z. Khayrullin, Vestnik MGSU, 13, 71 (2018)

7. R.Z. Khayrullin, Science Review, 18, 131 (2017) 